\title{
Coulomb effects in nuclear collisions
}

\author{
M. Bawin and J. Cugnon \\ Institut de Physique, Université de Liége, Sart Tilman, B-4000 Liége 1, Belgium
}

(Received 9 July 1981)

\begin{abstract}
We compare the analytic approximation to the Coulomb phase space factor recently given by Gyulassy and Kauffmann to the expression obtained from an exact solution of the Klein-Gordon equation. We find that while the agreement is excellent for pions in field of small $Z(\mathrm{Ne})$ nuclei, a difference in magnitude of order $10^{2}$ may occur for low energy pions in high $Z(\mathrm{~Pb})$ fields, thus suggesting that an exact solution to the KleinGordon equation may be useful in the analysis of Coulomb effects in nuclear collisions of heavy elements.
\end{abstract}

NUCLEAR REACTIONS Coulomb final state interaction in pion] production. Exact solution of Klein-Gordon equation. Comparison with simple approximation.

Gyulassy and Kauffmann ${ }^{1}$ recently gave simple analytic formulas to study Coulomb final state interactions in a relativistic field theory model, which they applied to study recent data on nuclear collisions involving production of $\pi^{+}$and $\pi^{-}$particles. Specifically, if one writes the charged pion inclusive cross section $\sigma_{ \pm}(\overrightarrow{\mathrm{p}})$ in the form

$$
\sigma_{ \pm}(\overrightarrow{\mathrm{p}})=\sigma_{0}(\overrightarrow{\mathrm{p}} \pm \delta \overrightarrow{\mathrm{p}}) D_{ \pm}(\overrightarrow{\mathrm{p}}),
$$

where $\sigma_{0}(\overrightarrow{\mathrm{p}})$ is the corresponding cross section for neutral pions, $\delta \overrightarrow{\mathrm{p}}$ is the momentum shift due to the Coulomb field, and $D(\overrightarrow{\mathrm{p}})$ is the Coulomb phase space factor, the authors of Ref. 1 propose the following form for $D(\overrightarrow{\mathrm{p}})$ :

$$
D_{ \pm}(\overrightarrow{\mathrm{p}})=G\left( \pm \frac{\delta D}{\pi}\right)
$$

in the case of a single nuclear charge $Z$. In this expression, $G$ is the usual Gamow factor

$$
G(x)=\frac{2 \pi x}{e^{2 \pi x}-1},
$$

while

$$
\delta D=\pi \frac{\alpha Z E}{p} F(p),
$$

with

$$
p \equiv|\overrightarrow{\mathrm{p}}| \text {. }
$$

In these equations, $E$ and $\overrightarrow{\mathrm{p}}$ are the energy and momentum of the produced particle, $\alpha$ is the fine structure constant, and $F(p)$ takes the finite size of the nucleus into account. For an exponential charge distribution of radius $R$, this quantity is given by ${ }^{1}$

$$
F(p)=\frac{2}{\pi} \tan ^{-1} \frac{1}{p R}-\frac{1}{\pi} \frac{p R}{1+(p R)^{2}} .
$$

Equations (2) - (5) are valid in the frame of a single static nuclear charge. ${ }^{2}$ The authors of Ref. 1 expect formulas $(2)-(5)$ to provide a good approximation to the exact result

$$
D(\overrightarrow{\mathrm{p}})=|\psi(0)|^{2}
$$

for a wide range of the physical parameters. In this expression, $\psi(\vec{r})$ is a solution of the KleinGordon equation, normalized to an incident plane wave $e^{i k z}$ :

$$
\left[(E-V)^{2}-\overrightarrow{\mathrm{p}}^{2}-m^{2}\right] \psi(\overrightarrow{\mathrm{r}})=0
$$

where $V$ is the nuclear Coulomb field and $m$ is the mass of the charged particle. Furthermore, according to Ref. 1, the exact form of $F(p)$ is not crucial; what matters is that it has the same asymptotic behavior when $p \rightarrow \infty$ and $R \rightarrow 0$ as the one arising, for instance, from a static potential $V(r)$ of the form 


$$
\begin{array}{ll}
V(r)=\frac{\alpha Z}{r}, & r>R, \\
V(r)=\frac{\alpha Z}{R}, & r \leq R .
\end{array}
$$

As it would be useful indeed to have "pocket formulas" for the production cross section $\sigma_{ \pm}(\vec{p})$, we wish to study here in what range of the parameters $\eta=\alpha Z E / p$ and $\rho=p R$ expressions (2)-(5) provide a good approximation to the exact solution obtained by solving the Klein-Gordon equation (7) with the interaction (8).

We therefore solved the $s$-wave radial KleinGordon equation [other partial waves give a vanishing contribution to $\psi(0)]$ :

$$
\left\{\frac{d^{2}}{d r^{2}}+[E-V(r)]^{2}-m^{2}\right\} u(r)=0 .
$$

The procedure is quite standard. We just give the final result

$$
\begin{aligned}
u(r)=e^{k(\pi / 2)+i(\pi / 4)}[ & A \frac{e^{i(m \pi / 2)}}{\Gamma(1+2 m)} M_{i k, m}\left(-i \rho^{\prime}\right) \\
& \left.+B \frac{e^{-i(m \pi / 2)}}{\Gamma(1-2 m)} M_{i k,-m}\left(-i \rho^{\prime}\right)\right],
\end{aligned}
$$

for $r>R$, and

$$
u(r)=C \sin K r
$$

for $r<R . M_{k, m}(z)$ is the Whittaker function ${ }^{3}$ and

$$
\begin{aligned}
& \rho^{\prime}=2 p r \\
& m=\left(\frac{1}{4}-\alpha^{2} Z^{2}\right)^{1 / 2}, \\
& k=E \alpha Z\left(E^{2}-m^{2}\right)^{-1 / 2} \equiv \eta, \\
& K^{2}=\left(E-\frac{\alpha Z}{R}\right)^{2}-m^{2} .
\end{aligned}
$$

The coefficients $A, B$, and $C$ have been determined by requiring that the wave function $u(r)$ and its derivatives be continuous at $r=R$ and that

$$
\lim _{r \rightarrow \infty} u(r) \sim e^{-i\left(p r+\eta \log \rho^{\prime}\right)}+f(E) e^{i\left(p r+\eta \log \rho^{\prime}\right)} .
$$

Note that, since ${ }^{3}$

$$
M_{i k, m}(\rho) \underset{\rho \rightarrow 0}{\sim} \rho^{m+1 / 2},
$$

it follows that, in the point charge limit, the full radial wave function $\psi(r) \equiv u(r) / r$ diverges at $r=0$, in contrast with the corresponding result in the nonrelativistic case, which is finite.

We show in Fig. 1 the results of our calculation for $|\psi(r=0)|^{2}$ in the $\eta-\rho$ plane, as curves of equal value. The long-dashed curves correspond to a $\pi^{+}$in the field of a Ne nucleus (lower curve) and of a $\mathrm{Pb}$ nucleus (upper curve) and, in both cases, to an energy range extending from 3 to $300 \mathrm{MeV}$ kinetic energy. The curves have been obtained by considering several nuclei from $\mathrm{Ne}$ to $\mathrm{Pb}$, with, in each case, several radii between the ordinary radius and $\sim 1.4$ times this value. Larger radii have some interest in relativistic nuclear collisions, as the nuclear system is expanding. ${ }^{1,4,5}$

The small-dashed curves delineate the physical domain in the $(\eta-\rho)$ plane, considering the possible charges, radii, and the minimum value of detectable kinetic energy for a pion. We think the curves will be useful to experimentalists to analyze their pion production data. Let us furthermore mention that the programming code requires quite

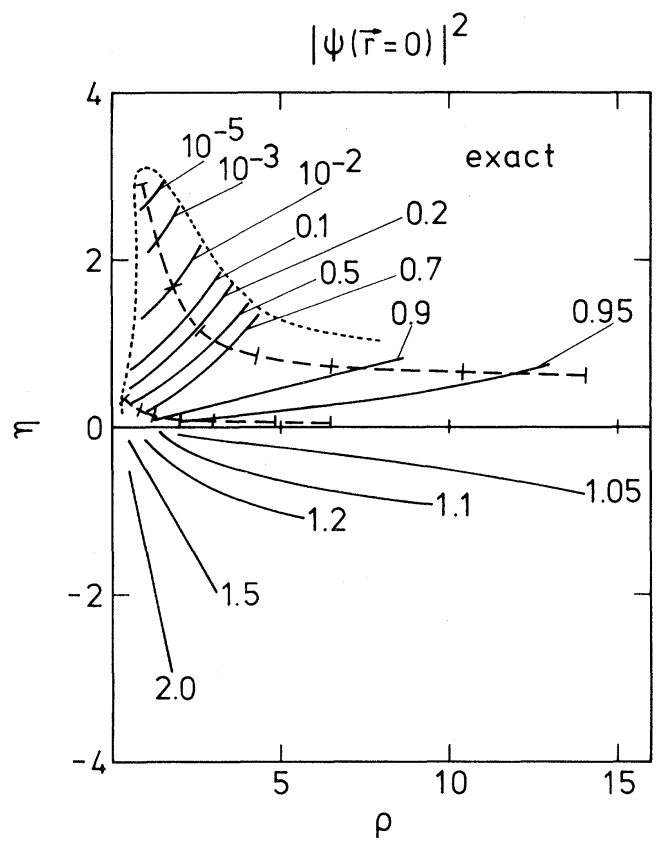

FIG. 1. Curves of equal value of the exact expression of $|\psi(r=0)|^{2}$ as calculated from Eqs. (9)-(16) for different values of the parameters $\eta=\alpha Z E p^{-1}$ and $\rho=p R$ (see text). The small dashes enclose the physical domain corresponding to detectable positive pions in usual nuclei. The long-dashed curves correspond to $\mathrm{Ne}$ (lower curve) and $\mathrm{Pb}$ (upper curve) cases, respectively. The little bars across these curves correspond to pion kinetic energies of $3,10,20,50,100,200$, and $300 \mathrm{MeV}$, successively, starting from the left. The lower part of the figure gives the result for negative pions. 
a small time $(\sim 0.15 \mathrm{sec}$ for one case on an IBM 370/158).

We wish, however, to emphasize that the KleinGordon equation we study here cannot describe the full complexity of the final state interaction even in a multicenter static Coulomb field. ${ }^{6}$ In addition, charges are even nonstatic in relativistic nucleus-nucleus collisions. Reference 1 provides acceptable prescriptions to deal with such a nonstatic situation. Our purpose here is merely to provide a better description of the Coulomb phase factor $D_{ \pm}(\overrightarrow{\mathrm{p}})$ [Eq. (1)], whose importance extends beyond heavy-ion physics. For instance, Eq. (1) applies to $(N, \pi)$ reactions as well.

In Fig. 2, we also show, in the $\eta-\rho$ plane, the ratio between the exact value of $|\psi(0)|^{2}$ and the approximations $(2)-(5)$ of Ref. 1 . We notice that in the case of $\mathrm{Ne}$, formulas (2) - (5) provide a good approximation to the exact wave function at the origin, as long as $\rho$ is not too close to zero. For heavier elements, however, we see that the approximation of Ref. 1 may lead to large deviations from the exact result for small energy. This is in agreement with the discussion of the validity of Eqs. (2)-(5) given in Ref. 1. In more general terms, the discrepancy appears in the "quantum domain" defined in Ref. 1, i.e., for

$$
2 \eta>\rho-\frac{1}{\rho} .
$$

We therefore conclude that exact wave functions should be useful in analyzing Coulomb effects coming from large Coulomb fields. In the context

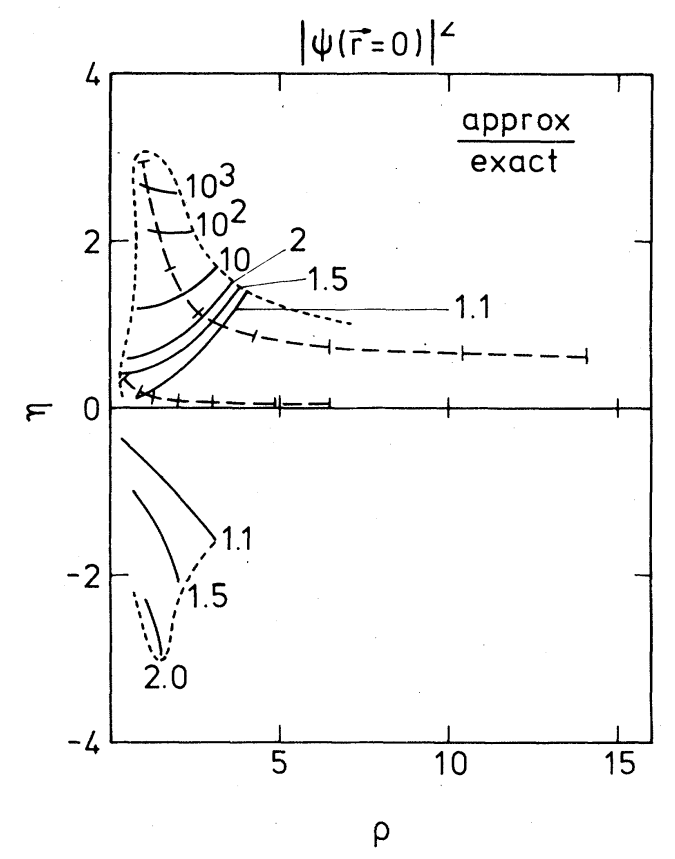

FIG. 2. Ratio of the approximated value proposed in Ref. 1 to the exact value of $|\psi(\vec{r}=0)|^{2}$ as calculated from Eqs. (9)-(16). The same convention as in Fig. 1 has been used.

of relativistic nuclear collisions, this remark especially applies to pions in the mid-rapidity region.

The work of one of us (M. B.) was supported by the National Fund for Scientific Research (Belgium).
${ }^{1}$ M. Gyulassy and S. K. Kauffmann, Nucl. Phys. A362, 524 (1981).

${ }^{2}$ Prescriptions are given in Ref. 1 to handle expanding charges.

${ }^{3}$ M. Abramowitz and I. A. Stegun, Handbook of Mathematical Functions (Dover, New York, 1970), Chap. 13.

${ }^{4}$ P. J. Siemens and J. O. Rasmussen, Phys. Rev. Lett. 42, 880 (1979).
5J. Cugnon and S. E. Koonin, Nucl. Phys. A355, 477 (1981).

${ }^{6}$ For a study of the complexity of scattering from a multicenter static field, see, e.g., P. G. Burke, Advances in Atomic and Molecular Physics (Academic, New York, 1968), Vol. IV, p. 173; N. F. Mott and H. S. W.

Massey, The Theory of Atomic Collisions (Clarendon, Oxford, 1965), Chap. 8. 